\title{
Rat Palatability Study for Taste Assessment of Caffeine Citrate Formulation Prepared via Hot-Melt Extrusion Technology
}

\author{
Roshan V. Tiwari, ${ }^{1}$ Ashley N. Polk, ${ }^{2}$ Hemlata Patil, ${ }^{1}$ Xingyou Ye, ${ }^{1}$ \\ Manjeet B. Pimparade, ${ }^{1}$ and Michael A. Repka ${ }^{1,3,4}$
}

Received 2 September 2015; accepted 4 November 2015; published online 16 November 2015

\begin{abstract}
Developing a pediatric oral formulation with an age-appropriate dosage form and taste masking of naturally bitter active pharmaceutical ingredients (APIs) are key challenges for formulation scientists. Several techniques are used for taste masking of bitter APIs to improve formulation palatability; however, not all the techniques are applicable to pediatric dosage forms because of the limitations on the kind and concentration of the excipients that can be used. Hot-melt extrusion (HME) technology is used successfully for taste masking of bitter APIs and overcomes some of the limitations of the existing taste-masking techniques. Likewise, analytical taste assessment is an important quality control parameter evaluated by several in vivo and in vitro methods, such as the human taste panel, electrophysiological methods, electronic sensor, and animal preference tests to aid in selecting a taste-masked formulation. However, the most appropriate in vivo method to assess the taste-masking efficacy of pediatric formulations remains unknown because it is not known to what extent the human taste panel/electronic tongue can predict the palatability in the pediatric patients. The purpose of this study was to develop taste-masked caffeine citrate extrudates via HME and to demonstrate the wide applicability of a single bottle-test rat model to record and compare the volume consumed of the taste-masked solutions to that of the pure API. Thus, this rat model can be considered as a low-cost alternative taste-assessment method to the most commonly used expensive human taste panel/electronic tongue method for pediatric formulations.
\end{abstract}

KEYWORDS: caffeine citrate; hot-melt extrusion; pediatric; rat model (single bottle test); taste masking.

\section{INTRODUCTION}

Oral drug delivery is the most convenient and commonly employed route of drug administration for all age populations. The preference of the oral dosage form over other dosage forms is due to various factors, such as its non-invasive nature, ease-of-administration, and patient compliance. However, many active pharmaceutical ingredients (APIs) are naturally bitter, which is a real challenge for pharmaceutical scientists in developing a palatable formulation to be used for infants and children. Drug taste is one of the most important factors that determine the palatability/acceptability of the oral dosage form and, thus, patient compliance, especially concerning the

\footnotetext{
${ }^{1}$ Department of Pharmaceutics and Drug Delivery, School of Pharmacy, The University of Mississippi, University, Mississippi 38677, USA.

${ }^{2}$ Department of Psychology, The University of Mississippi, University, Mississippi 38677, USA.

${ }^{3}$ Pii Center for Pharmaceutical Technology, School of Pharmacy, The University of Mississippi, University, Mississippi 38677, USA.

${ }^{4}$ To whom correspondence should be addressed. (e-mail: marepka@olemiss.edu)
}

pediatric and geriatric population. Therefore, pharmaceutical scientists have developed different techniques to mask bittertasting APIs in oral dosage formulations. The commonly used taste-masking techniques are the organoleptic method, which uses a sweetener and/or flavors, complexation, coating, matrix entrapment, prodrug formation, spray drying, and salt formation $(1,2)$. Studies have shown that the hot-melt extrusion (HME) technology can also be used effectively for the taste masking of bitter APIs (3-6). The advantage of HME is that it is a continuous, solvent-free process, easy to scale-up, and has a short processing time, and several of the polymer carrier and other additives used during the extrusion processing are generally known to be safe for consumption (7).

Along with taste masking, taste evaluation is an important quality parameter for the evaluation of taste-masked formulations. For the in vivo taste assessment in humans or animals, the taste stimuli (test sample) should come into contact with the taste buds on the tongue. This initiates the interaction of the test sample with the receptors on the taste cells embedded in the membrane of the taste buds. The taste information is then transmitted to the nerve fiber endings, which send the impulses along the cranial nerves to the brain where the taste is perceived (8). Such in vivo assessments include human taste panels, an electronic tongue, 
electrophysiological methods, and animal preference tests $(9,10)$. Among these commonly used taste assessment methods, human taste panel studies are significantly influenced by age-related differences, i.e., the differences in the response to taste stimuli between adults and children. Thus, the human/adult taste panel studies used for predicating the taste of pediatric formulations is debatable. The other method is the electronic tongue (in vitro), which can assess taste via an analytical multichannel taste-sensing sensory system; however, the use of the electronic tongue has thus far not been validated for pediatric taste perception.

As an alternative to the human taste panel and electronic tongue, taste-masked formulations can be evaluated in animals in vivo. The two-bottle preference test, conditioned taste aversion, and monitoring of the licking frequency ("the Davis rig") are commonly used methods to determine the taste preference and concentration-response properties of taste stimuli by animals (8,11-13). Rats, mice, cats, and dogs are commonly used for such taste preference tests. It has been previously reported that rodents (rats and mice) are particularly useful for taste perception studies as they share several behaviors and physiological characteristics with humans, for example, similar taste sensitivities (14). Very young children share some other similarities with rodents, such as the identification of taste perception through behavior rather than through language (15).

In the present study, caffeine citrate (CC, 2hydroxypropane-1,2,3-tricarboxylic acid; 1,3,7trimethylpurine-2,6-dione, Fig. 1) was chosen as a model API to evaluate the taste-masking efficacy. $\mathrm{CC}$ is an odorless, bitter-tasting white crystalline powder. Caffeine is used therapeutically to treat episodes of apnea in premature infants (16). Since $\mathrm{CC}$ is a highly soluble Biopharmaceutics Classification System (BCS) class-I drug, its rapid dissolution in the oral cavity impedes its taste masking (17). Considering all of these physicochemical properties, taste-masked CC extrudates were developed via HME technology to improve patient acceptance and compliance of CC. The authors have used in vitro and in vivo methods to evaluate the efficacy of the HME process in masking the unpleasant bitter taste of CC. The primary aim of this study was to develop a nonhuman, non-electronic, low-cost, and single bottle-test rat model for the assessment of taste-masked formulations. Furthermore, the authors aimed to establish this model for the future evaluation of bitter API taste-masking formulations, especially in the development of pediatric formulations.

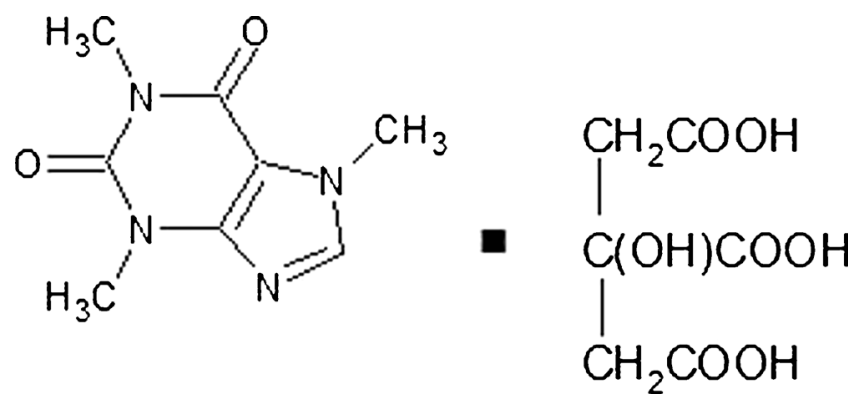

Fig. 1. Chemical structure of caffeine citrate

\section{MATERIALS AND METHODS}

\section{Materials}

CC was purchased from Fisher Scientific (Pittsburgh, PA, USA). Neosorb ${ }^{\circledR}$ (Sorbitol) was purchased from Roquette Pharma (USA). Compritol ${ }^{\circledR} 888$ ATO (Glyceryl Dibehenate EP) was a kind gift from Gattefossé (Paramus, NJ, USA). Kollidone ${ }^{\circledR}$ VA 64 was a gift sample from BASF (Florham Park, NJ, USA). Eudragit@ EPO was supplied by Evonik (USA). D-fructose was purchased from Fisher Scientific (Fair Lawn, NJ, USA). All of the other reagents, such as methanol (impurities $0.1 \%$ ), used in this study were of analytical grade.

\section{Methods}

\section{Hot-Melt Extrusion Processing}

Prior to HME processing, the excipients were passed through an ASTM \#30-mesh screen to remove any aggregates that might have been formed. The constituents of all formulations shown in Table I were mixed using a V-shell blender (Maxiblend®; GlobePharma, New Brunswick, NJ, USA) at $20 \mathrm{rpm}$ for $20 \mathrm{~min}$. The physical blends were subsequently transferred to the feeder of a co-rotating twin-screw extruder (Haake MiniLab, Thermo Electron; Newington, NH, USA). The screw speed was set at $100 \mathrm{rpm}$ for all four formulations (F1-F4). The heating temperature for F1-F4 was 84, 70, 150, and $130^{\circ} \mathrm{C}$, respectively. The extrudates were milled using a comminuting mill (Fitzpatrick, Model "L1A"; Elmhurst, IL, USA). The milled extrudates were sieved to a particle size ranging from 300 to $425 \mu \mathrm{m}$ using the extrudates retained between the US \#35 and US \#40 sieves. The extrudates were stored in foil-lined polyethylene bags until further analysis and processing.

\section{Thermal Gravimetric Analysis}

Thermal gravimetric analysis (TGA) studies were performed on a Perkin Elmer Pyris 1 TGA with the Pyris software to determine the thermal stability of $\mathrm{CC}$ and the excipient during the HME processing. Samples weighing 5$8 \mathrm{mg}$ were heated at a heating rate of $40^{\circ} \mathrm{C} / \mathrm{min}$ from $50^{\circ} \mathrm{C}$ to $320^{\circ} \mathrm{C}$ in a platinum pan under an inert nitrogen atmosphere purge of $20 \mathrm{~mL} / \mathrm{min}$.

\section{Differential Scanning Calorimetry}

Differential scanning calorimetry (DSC) studies were performed with a Perkin Elmer Diamond differential scanning calorimeter (Shelton, CT, USA) to measure the degree of crystallinity of the samples. Data analysis was performed with the Pyris manager software. About 2-5 mg of the samples were weighed and sealed hermetically in an aluminum pan. The heating rate was set at $20^{\circ} \mathrm{C} / \mathrm{min}$ from $20^{\circ} \mathrm{C}$ to $250^{\circ} \mathrm{C}$ under an inert atmosphere of nitrogen at a flow rate of $20 \mathrm{~mL} / \mathrm{min}$. 
Table I. Formulation composition

\begin{tabular}{lllll}
\hline Formulation $(\%)$ & F1 & F2 & F3 & F4 \\
\hline Caffeine citrate & 33.33 & 33.33 & 33.33 & 33.33 \\
Neosorb® & 66.67 & - & - & - \\
Compritol® ATO 888 & - & 66.67 & - & - \\
Kollidone® VA 64 & - & - & 66.67 & - \\
Eudragit® EPO & - & - & - & 66.67
\end{tabular}

Post-Processing Caffeine Citrate Content and Content Uniformity

The extrudates were first milled to a fine powder. The resultant powders were accurately weighed and were dissolved in methanol to water 1:1, diluted, and filtered using $0.2-\mu \mathrm{m}, 13-\mathrm{mm}$ PTFE membrane filters (Whatman, Piscataway, NJ). The samples were subsequently analyzed by UV-vis spectrophotometry. The extrudates were also evaluated for content uniformity. Briefly, 20 different samples from the milled extrudates were selected randomly and were triturated using a mortar and pestle. The resultant powders were accurately weighed and were dissolved in methanol to water $1: 1$, and the CC content was determined using UV-vis spectrophotometry.

\section{$U V$-Vis Spectrophotometric Analysis}

The samples were analyzed using a GENESYS 6 UV-vis spectrophotometer (Thermo Scientific, Madison, WI, US) at a wavelength of $273 \mathrm{~nm}$. The standard curve was linear over the range of $5-30 \mu \mathrm{g} / \mathrm{mL}$ with $R^{2}$ equal to 0.998 .

\section{In vitro Dissolution}

The milled and sieved extrudates were tested for their in vitro $\mathrm{CC}$ release capacity in two dissolution media: artificial saliva (SSF, pH 6.8, Table II) and ultra-purified water. The first dissolution study was performed in $500 \mathrm{~mL}$ of SSF adjusted to $\mathrm{pH} 6.8$ for oral $\mathrm{CC}$ release using an USP apparatus II (Hanson SR8-plus ${ }^{\mathrm{TM}}$; Hanson, Chatsworth, CA, US) set at 37 $\pm 0.5^{\circ} \mathrm{C}$ with a rotation speed of $50 \mathrm{rpm}$ and equipped with UV-vis probes (Rainbow Dissolution Monitor; Pion, Inc., Billerica, MA, USA). Spectra were collected at $273 \mathrm{~nm}$ every $5 \mathrm{~s}$ for a total of $30 \mathrm{~s}$. The second dissolution study was performed in $900 \mathrm{~mL}$ of ultra-purified water. The SR8-plus ${ }^{\mathrm{TM}}$ dissolution apparatus II was maintained at $37 \pm 0.5^{\circ} \mathrm{C}$, and the paddle speed was set at $50 \mathrm{rpm}$. Milled extrudates equivalent to $100 \mathrm{mg}$ of $\mathrm{CC}$ were used for the dissolution study. The samples were collected at intervals of 10, 20, 40, and $60 \mathrm{~min}$

Table II. Artificial saliva dissolution medium (adjusted to $\mathrm{pH}$ 6.8)

\begin{tabular}{lc}
\hline Compound & Concentration $(\mathrm{g} / \mathrm{L})$ \\
\hline $\mathrm{CaCl}_{2} \cdot 2 \mathrm{H}_{2} \mathrm{O}$ & 0.228 \\
$\mathrm{MgCl}_{2} \cdot 6 \mathrm{H}_{2} \mathrm{O}$ & 0.061 \\
$\mathrm{NaCl}$ & 1.017 \\
$\mathrm{~K}_{2} \mathrm{CO}_{3} \cdot 1.5 \mathrm{H}_{2} \mathrm{O}$ & 0.603 \\
$\mathrm{Na}_{2} \mathrm{HPO}_{4} \cdot 7 \mathrm{H}_{2} \mathrm{O}$ & 0.204 \\
$\mathrm{NaH}_{2} \mathrm{PO}_{4} \cdot \mathrm{H}_{2} \mathrm{O}$ & 0.273 \\
\hline
\end{tabular}

through a stainless steel cannula with a $0.2-\mu \mathrm{m}$ nylon filter tip attached to a $2.5-\mathrm{mL}$ syringe. The samples were analyzed directly using a UV-vis spectrophotometer set at $273 \mathrm{~nm}$.

\section{In vivo Taste Assessment}

Animals. Twenty-one naïve adult male Sprague-Dawley rats (175-200 g) (Harlan Laboratories, Inc., Houston, TX, USA) were used for the study. Two rats each were housed in Plexiglas cages with corncob bedding in a vivarium that maintained a 12-h light/dark cycle and an ambient temperature of $\sim 22^{\circ} \mathrm{C}$. Food and water were available ad libitum except during the training and testing water restriction conditions as mentioned below. All procedures were approved by the Institutional Animal Care and Use Committee (IACUC) at The University of Mississippi, Oxford, USA (protocol no. 15-026).

Training paradigm. The rats were deprived of water for a period of $22 \mathrm{~h}$ to motivate the licking behavior (response to thirst) but had access to food ad libitum. After the water deprivation period, the Plexiglas cage was divided into compartments by aid of a divider. Subsequently, the rats were provided free access to $50 \mathrm{~mL}$ of water in a graduated drinking bottle for a period of $30 \mathrm{~min}$, followed by removal of the bottles and recording of the volume consumed. This training paradigm continued for 2 days before the actual start of the experiments. The rats were subsequently divided into 3 groups ( $n=7 /$ group).

Experiment. A 5-day experiment was conducted. The experimental period lasted $30 \mathrm{~min}$ following the 22 -h water deprivation period after which the rats had free access to water for $1.5 \mathrm{~h}$ before the start of the 22 -h water deprivation period for the next day experiment. The rats in all groups had access to food ad libitum. During the 30-min experimental period, all groups had free access to $50 \mathrm{~mL}$ of the test solutions in a graduated drinking bottle according to the schedule below.

Day 1: water

Day 2: fructose-water solution $(0.5 \mathrm{~g} / \mathrm{L})$

Day 3: Group 1-CC (CC, $1 \mathrm{mg} / \mathrm{mL})$, group 2-CC (CC, $2 \mathrm{mg}$ / $\mathrm{mL})$, and group 3-CC (CC, $4 \mathrm{mg} / \mathrm{mL})$

Day 4: water

Day 5: Group 1-F1 (CC, $4 \mathrm{mg} / \mathrm{mL})$, group 2-F3 (CC, $4 \mathrm{mg}$ / $\mathrm{mL})$, and group 3-F4 (CC, $4 \mathrm{mg} / \mathrm{mL})$

At the end of the 30-min experiment period, the bottles were removed and the volume consumed was recorded. The volume recorded minus any loss in the volume of the test solution due to keeping or removing the bottle was considered 
a correction factor in the record of the volume consumed. Other behavior responses to the bitter-taste stimuli (test solution), such as jaw smacking, oral grooming, and retreating, were also observed but not analyzed for this particular study.

\section{Statistical Analysis}

The differences between the groups were analyzed by one-way analysis of variance (ANOVA) followed by Dunnett's $t$ test or Tukey's multiple comparison test. A difference of $P<0.05$ was considered statistically significant as compared to the groups defined in the Figure legends.

\section{Results and Discussion}

\section{Thermogravimetric Analysis}

TGA studies were performed on CC and all four excipients. The TGA thermograms of the samples were observed for changes in weight. The TGA thermograms as shown in Fig. 2 indicated that all four excipients were stable up to $320^{\circ} \mathrm{C}$. Degradation was observed for $\mathrm{CC}>250^{\circ} \mathrm{C}$. These results indicated that the employed extrusion temperature range $\left(<200^{\circ} \mathrm{C}\right)$ did not result in the degradation of the formulations.

\section{Differential Scanning Calorimetry}

Prior to HME processing, analysis of the thermal behavior of an API and the excipients is critical as degradation of the ingredients or incompatibility due to the chemical reaction between the API and the excipients can be induced thermally. DSC studies were performed to investigate the physical state of $\mathrm{CC}$, the excipients, and the physical state of $\mathrm{CC}$ within the polymer matrix after extrusion. As shown in Fig. 3, pure CC exhibited a sharp endothermic peak onset at $168^{\circ} \mathrm{C}$ whereas this occurred at $100^{\circ} \mathrm{C}$ and $78^{\circ} \mathrm{C}$ for Neosorb and Compritol ATO 888, respectively. The DSC thermogram of Kollidon ${ }^{\circledR}$ VA 64 and Eudragit ${ }^{\circledR}$ EPO showed the absence of an endothermic melting peak, confirming the amorphous nature of these two polymers. The DSC thermogram of F1-F4 showed that the endothermic melting peak at around $168^{\circ} \mathrm{C}$ was present in $\mathrm{F} 2$, indicating that most of the $\mathrm{CC}$ in $\mathrm{F} 2$ remained in the crystalline state after extrusion. However, CC's endothermic melting peak was absent in the extrudates in F1, F3, and F4, indicating the transformation of CC from the crystalline to the amorphous state. This change in the physical state of $\mathrm{CC}$ within the extrudates was due to the different temperature employed during the extrusion process: the lowest temperature $\left(70^{\circ} \mathrm{C}\right)$ was used for $\mathrm{F} 2$, whereas the temperature for the other formulations was $>80^{\circ} \mathrm{C}$.

\section{Post-Processing Caffeine Citrate Content and Content Uniformity}

The CC content and content uniformity in the prepared extrudates were determined. All of the formulations possessed exceptional CC content (>98\%) as well as content uniformities $(<2 \% \mathrm{RSD})$ after the extrusion process, indicative of a robust formulation and processing method.

\section{In vitro Dissolution Study}

The in vitro dissolution study for F1-F4 was performed in SSF and ultra-purified water. Dissolution testing using SSF was used as a primary screening method for the tastemasking efficacy of the formulation. Figure 4 shows the $\mathrm{CC}$ release profile for formulations F1-F4 in SSF. Among all of the four formulations, F2 and F4 showed less than $3.5 \% \mathrm{CC}$ release in the first $30 \mathrm{~s}$ whereas $\mathrm{F} 1$ and $\mathrm{F} 3$ showed $8.7 \%$ and $5.0 \% \mathrm{CC}$ release, respectively, indicating that $\mathrm{F} 2$ and $\mathrm{F} 4$ could be promising candidate taste-masking formulations.

Figure 5 shows the in vitro $\mathrm{CC}$ release profile of $\mathrm{F} 1-\mathrm{F} 4$ in ultra-purified water. The results of the dissolution profiling varied among the tested formulations. F1, F3, and F4 showed a CC release of around 95\%, 93\%, and 99\%, respectively at the end of the $1-\mathrm{h}$ dissolution study, whereas only $12 \%$ CC was released in F2. This might have been due to the nature of the matrix carrier used in the

TGA

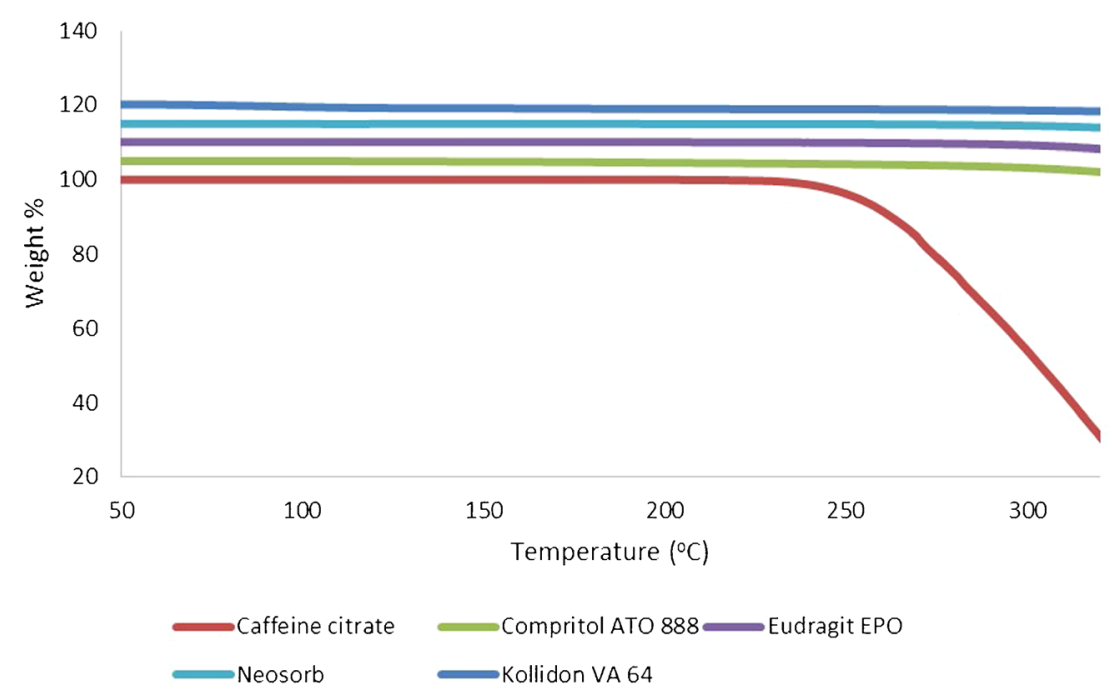

Fig. 2. Thermogravimetric analysis 


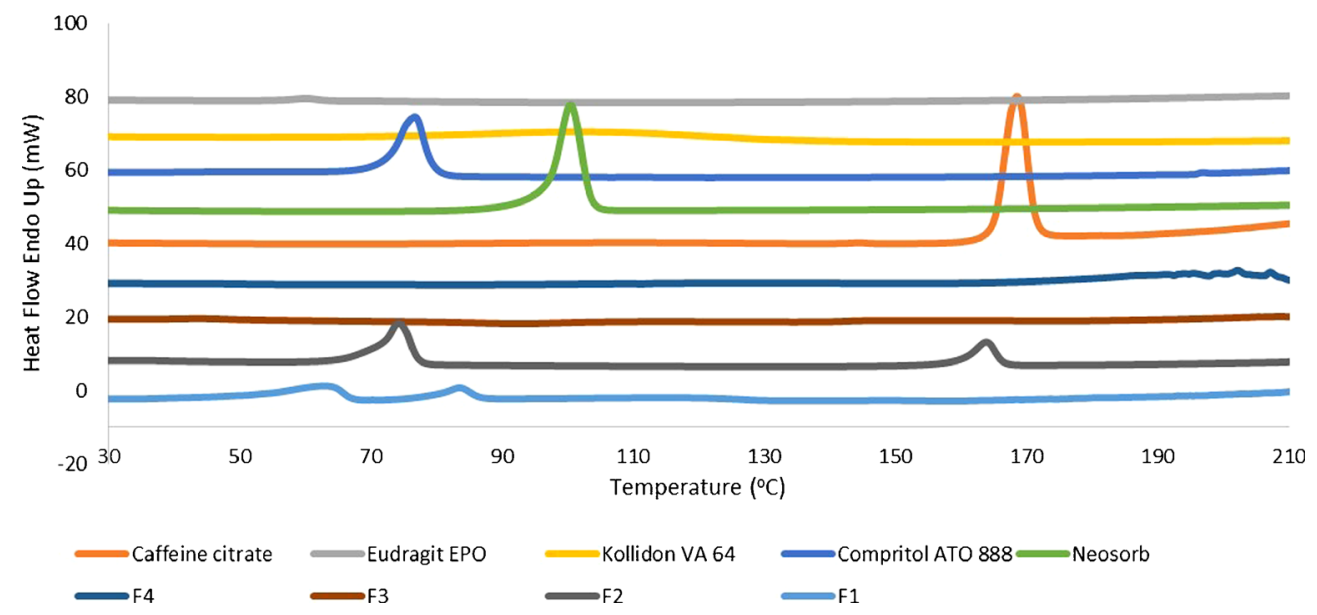

Fig. 3. Differential scanning calorimetry

extrudates. F1, F3, and F4 contained a hydrophilic matrix carrier resulting in the rapid dissolution of the tablet matrix and faster CC release, whereas F2 contained a lipophilic matrix, which is not water-soluble and the $\mathrm{CC}$ release was therefore much slower as compared to that of the other formulations. At the end of the first $10 \mathrm{~min}$ of the dissolution study, F1 containing Neosorb as matrix carrier dissolved very rapidly and showed therefore a $\mathrm{CC}$ release of $22.43 \%$. F3 and F4 showed only a $10.03 \%$ and $2.15 \%$ CC release, respectively. Very slight $\mathrm{CC}$ release was observed for formulation $\mathrm{F} 2$ at the end of the first $10 \mathrm{~min}$. All these results indicated that $\mathrm{F} 4$ could prevent $\mathrm{CC}$ release up to the first $10 \mathrm{~min}$ of the study (only $2.15 \%$ CC release) but that it had released around $99 \%$ of the $\mathrm{CC}$ at the end of the 1-h study. Therefore, F4 might be the most promising formulation to mask the bitter taste of $\mathrm{CC}$ as well as to achieve the highest $\mathrm{CC}$ release within an hour.

\section{In vivo Taste Assessment}

After $30 \mathrm{~min}$ of access to the test solutions, the total volume of the test solutions consumed was recorded (Fig. 6). On day 1, the total volume of water consumed at the end of the 30-min experiment was approximately $15 \mathrm{~mL}$, and neutral mouth movements were observed, indicating that the rats' taste perception of water was relatively neutral. On experimental day 2, a fructose-water solution was used as a sweet test solution. The total volume consumed at the end of the 30-min experiment was approximately $15 \mathrm{~mL}$, and a normal hedonic reaction was observed, i.e., rhythmic midline tongue protrusions and lateral tongue protrusions (discrete

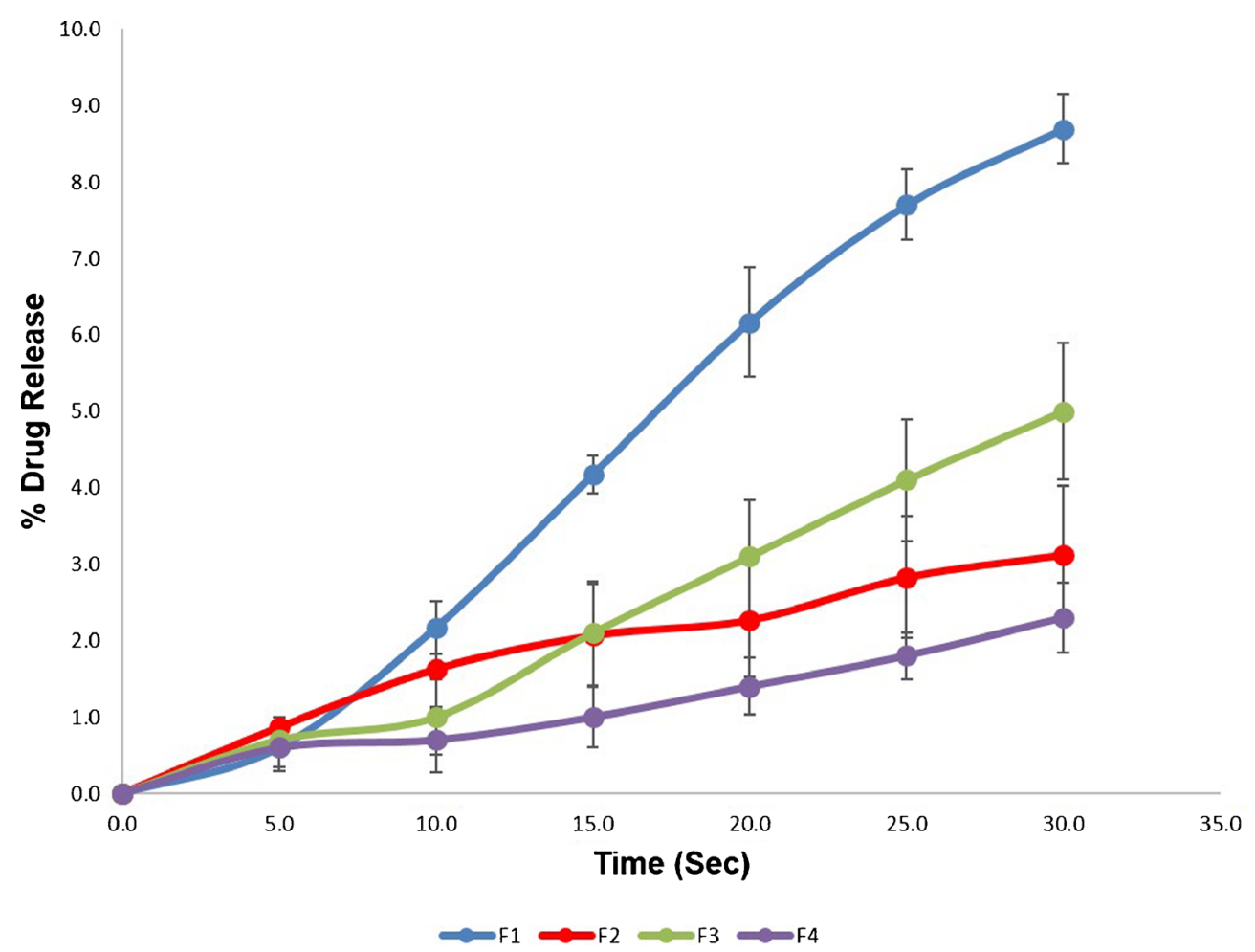

Fig. 4. In vitro drug release in artificial salivary media 


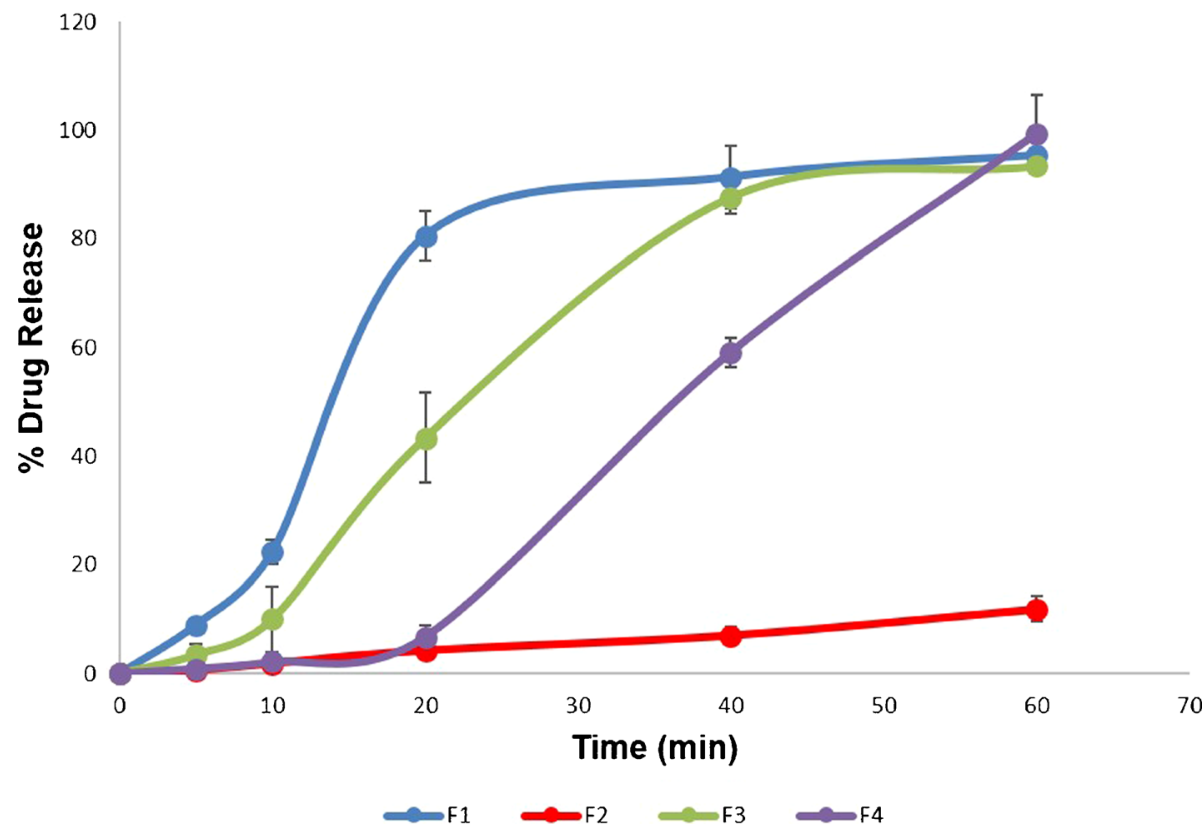

Fig. 5. In vitro drug release in ultra-purified water

and non-rhythmic) and paw licking (Fig. 7) (18). The volume of the fructose-water solution consumed and the rats' behavior indicated that the rats liked the taste of fructose. When the rats were provided free access to pure CC for $30 \mathrm{~min}$, a concentration-dependent decrease in the consumption of the test solution/pure CC was observed. Taste aversion behavior was observed as well, such as jaw smacking, oral grooming, nose wrinkle, paw wipe, forelimb flail, head shake, paw shakes, and retreating (Fig. 7) (19), indicating that the rats did not like the taste of pure $\mathrm{CC}$. This behavioral observation was more pronounced in the $3-\mathrm{CC}$ group $(4 \mathrm{mg} / \mathrm{mL})$ as the rats in this group had consumed significantly less $\mathrm{CC}$ solution by the end of the 30-min experiment as compared to the volume of water and fructose-water solution, further indicating that the rats did not like the taste of CC. Since rats have taste perception (memory), to prevent any false-positive or false-negative observations in the subsequent experiments, the rats were provided with

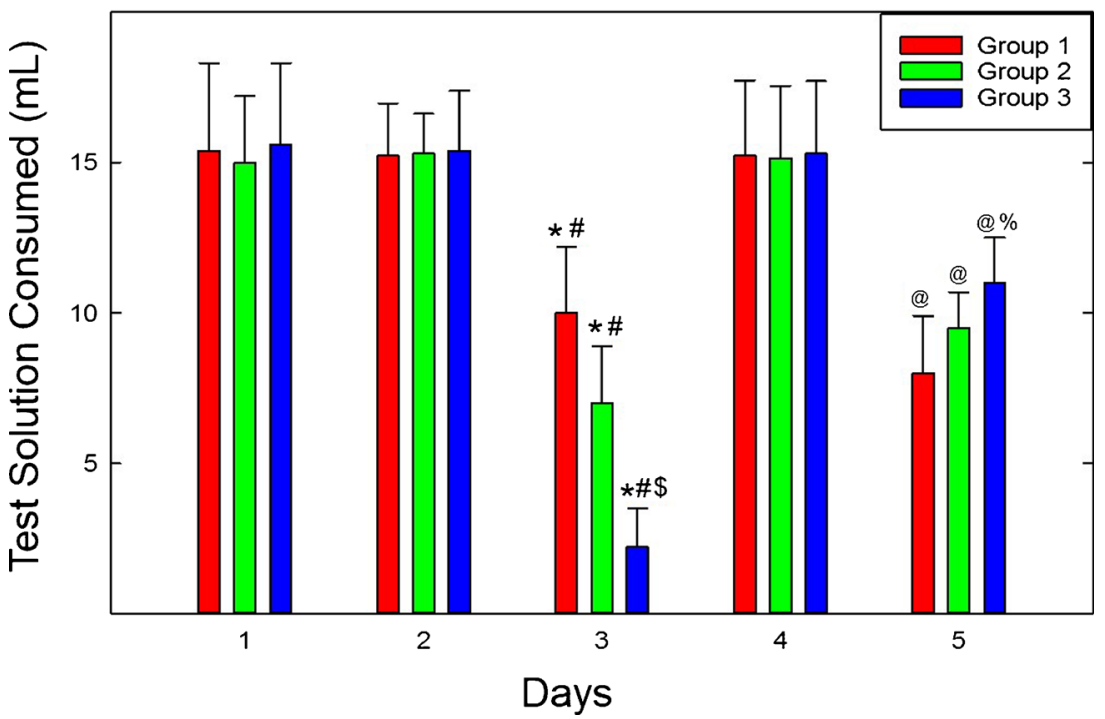

Fig. 6. In vivo taste assessment. $* P<0.05$, statistically significant difference in the volume of the test solution consumed (mL) as compared to that on day 1 (water). ${ }^{\#} P<0.05$, statistically significant difference in the volume of the test solution consumed $(\mathrm{mL})$ as compared to that on day 2 (fructose-water solution, $0.5 \mathrm{~g} / \mathrm{L}$ ). ${ }^{\$} P<0.05$, statistically significant difference in the volume of the test solution consumed $(\mathrm{mL})$ as compared to that of group 1-CC (pure caffeine citrate $(\mathrm{CC}), 1 \mathrm{mg} / \mathrm{mL}$ ) and group $2-\mathrm{CC}$ (pure $\mathrm{CC}, 2 \mathrm{mg} / \mathrm{mL}$ ) on day $3 .{ }^{\circledR} P<0.05$, statistically significant difference in the volume of the test solution consumed $(\mathrm{mL})$ as compared to that of group $3-\mathrm{CC}$ (pure CC, $4 \mathrm{mg} / \mathrm{mL}$ ) on day $3 .{ }^{\%} P<0.05$, statistically significant difference in the volume of the test solution consumed $(\mathrm{mL})$ as compared to that of group 1-F1 on day 5 


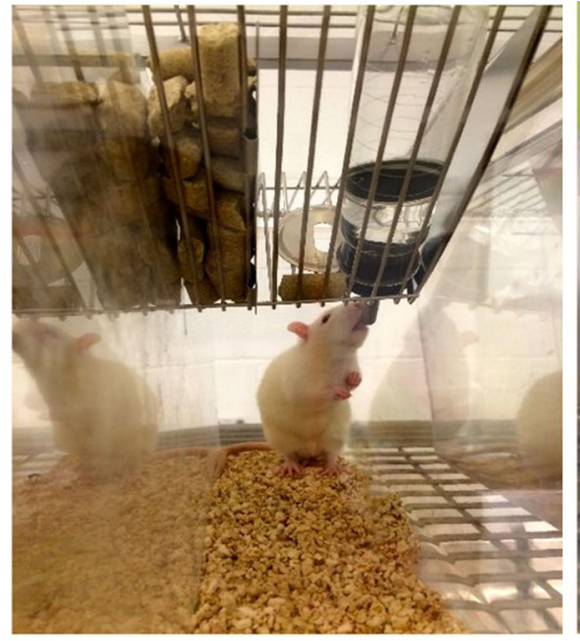

a Normal drinking

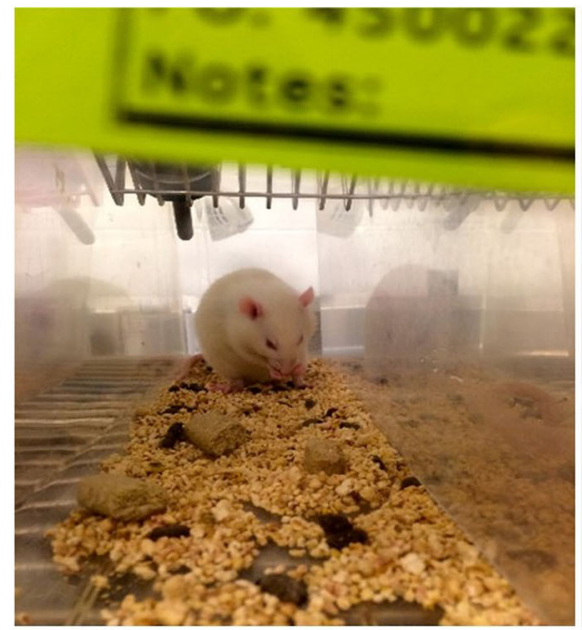

C Paw licking

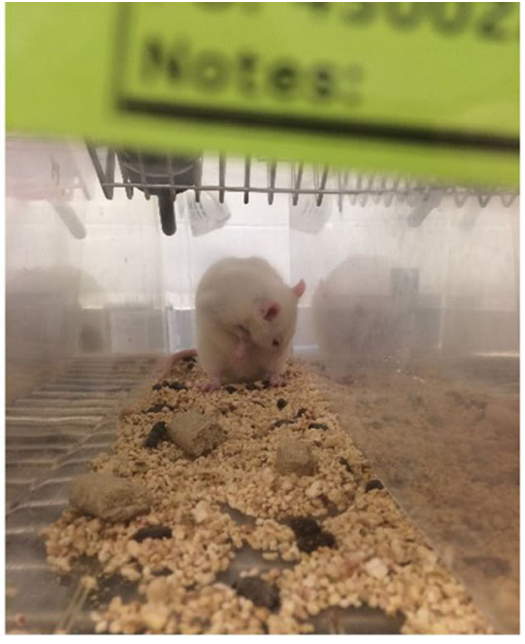

b Oral grooming

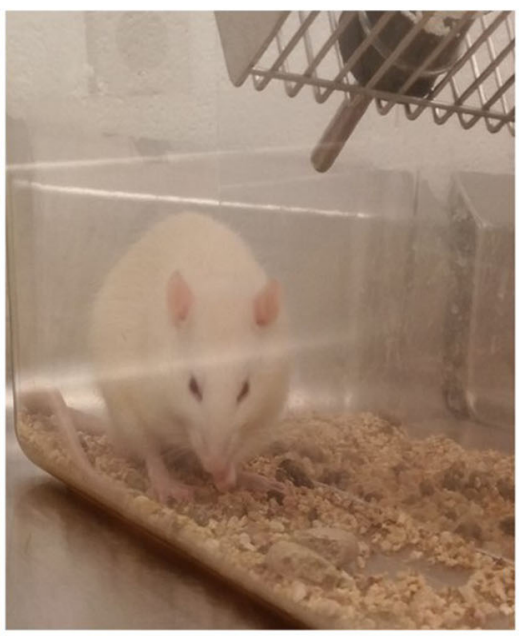

d Retreating

Fig. 7. Behavioral response of the rats during the taste assessment study

water again on day 4. On day 5, each group was provided with F1, $\mathrm{F} 3$, and F4. F2 was excluded from the in vivo test assessment experiment because of its low (11\%) CC release as observed at the end of the 1-h in vitro dissolution experiment. The results of the in vivo test assessment on day 5 showed a significant increase in the consumption of the test solutions at the end of the 30-min experiment as compared to that of the pure $\mathrm{CC}$ (4 $\mathrm{mg} / \mathrm{mL}$ ) solution, and the rats' behavior was neutral to slightly aversive tinge (in some rats a moderate nose wrinkle and head shake was observed). Thus, the volume consumed and the rats' behavior indicated that the bitter taste of $\mathrm{CC}$ was effectively masked in all three HME extrudates tested (F1, F3, and F4). Early literature on the behavioral pattern of human infants, primates, and rats has clearly demonstrated a correlation between animals and human infants in the facial expression and certain taste-related behaviors (e.g., tongue protrusion), which predict taste palatability or hedonic impact (pleasant taste) (18-21). This observation was further supported in this study by the higher consumption of the test solutions over that of pure $\mathrm{CC}$ by the rats, which indicated that the rats liked the taste of the test solutions'.

\section{In vitro Drug Release and In vivo Taste Assessment Correlation}

Our results showed a high correlation between the in vitro drug release in SSF and ultra-purified water dissolution medium and the in vivo taste assessment. Among all four formulations tested, F1 showed the highest CC release $(8.7 \%)$ within $30 \mathrm{~s}$ in SSF as well as in ultra-purified water dissolution medium after 10 and $30 \mathrm{~min}$. The in vivo taste assessment study results showed that the total volume consumed of F1 was approximately $8 \mathrm{~mL}$, which was the lowest volume consumed as compared to that of F3 and F4. This indicated that $\mathrm{CC}$ was released at a faster rate in $\mathrm{F} 1$ and that the bitter taste of F1 appeared faster than that of the other formulations. F4 showed the highest CC taste-masking efficacy with only $2.3 \%$ and $2.15 \% \mathrm{CC}$ release at the end of the 30-s experiment in SSF and at the end of the 10-min experiment in ultra-purified water dissolution medium, respectively. Furthermore, the total volume of F4 extrudate consumed was $11 \mathrm{~mL}$, which was higher than that consumed of F1 and F3, confirming that CC was not released at a faster rate and that it was successfully taste-masked. 


\section{CONCLUSION}

The well-known taste assessment methods used by pharmaceutical industries are beset by limitations, such as their expensive and time-consuming nature, ethical concerns regarding human taste panels, and the difficulty to correlate the taste perception of the human/adult taste panel with pediatric taste perception. Moreover, the validation of the electronic tongue for use in the pediatric population is questionable. The rat model used herein may be a valuable tool for the taste assessment of taste-masked formulations as it has considerable potential for screening of bitter APIs at each step of the development phase and the APIs' evaluation in the final commercial formulations. Along with the measurement of the test formulation volume consumed, this rat model has psychophysical taste perception characteristics (facial expression and/or tongue protrusion) similar to those observed in children. Therefore, this model may be employed as an effective alternative method to the human taste panel and the electronic tongue for the evaluation of the taste-masking efficacy of pediatric formulations containing bitter-tasting APIs.

\section{ACKNOWLEDGMENTS}

This project was partially supported by Grant Number P20GM104932 from the National Institute of General Medical Sciences (NIGMS), a component of NIH.

\section{COMPLIANCE WITH ETHICAL STANDARDS}

Ethical Approval All procedures were approved by the Institutional Animal Care and Use Committee (IACUC) at The University of Mississippi, Oxford, USA (protocol no. 15-026).

\section{REFERENCES}

1. Sohi H, Sultana Y, Khar RK. Taste masking technologies in oral pharmaceuticals: recent developments and approaches. Drug Dev Ind Pharm. 2004;30:429-48.

2. Preis M. Orally disintegrating films and mini-tablets-innovative dosage forms of choice for pediatric use. AAPS PharmSciTech. 2015;16(2):234-41.

3. Gryczke A, Schminke S, Maniruzzaman M, Beck J, Douroumis D. Development and evaluation of orally disintegrating tablets (ODTs) containing Ibuprofen granules prepared by hot melt extrusion. Colloids Surf B Biointerfaces. 2011;86:275-84.

4. Maniruzzaman M, Boateng JS, Bonnefille M, Aranyos A, Mitchell JC, Douroumis D. Taste masking of paracetamol by hot-melt extrusion: an in vitro and in vivo evaluation. Eur $\mathrm{J}$ Pharm Biopharm. 2012;80:433-42.

5. Alshehri SM, Park JB, Alsulays BB, Tiwari RV, Almutairy B, Alshetaili AS, et al. Mefenamic acid taste-masked oral disintegrating tablets with enhanced solubility via molecular interaction produced by hot melt extrusion technology. J Drug Deliv Sci Technol. 2015;27:18-27.

6. Pimparade MB, Morott JT, Park JB, Kulkarni VI, Majumdar S, Murthy SN, et al. Development of taste masked caffeine citrate formulations utilizing hot melt extrusion technology and in vitro-in vivo evaluations. Int J Pharm. 2015;487:167-76.

7. Patil H, Tiwari RV, Repka MA. Hot-melt extrusion: from theory to application in pharmaceutical formulation. AAPS PharmSciTech. 2015. doi:10.1208/s12249-015-0360-7.

8. Anand V, Kataria M, Kukkar V, Saharan V, Choudhury PK. The latest trends in the taste assessment of pharmaceuticals. Drug Discov Today. 2007;12:257-65.

9. Maniruzzaman M, Boateng JS, Chowdhry BZ, Snowden MJ, Douroumis D. A review on the taste masking of bitter APIs: hotmelt extrusion (HME) evaluation. Drug Dev Ind Pharm. 2014;40:145-56.

10. Preis M, Häusler O, Breitkreutz J. Electronic tongues in preformulation studies: application of electronic tongues in preformulation studies to evaluate taste-masking capacities of maltodextrins. Pharm Ind. 2014;76(6):957-62.

11. Tordoff MG, Bachmanov AA. Mouse taste preference tests: why only two bottles? Chem Senses. 2003;28:315-24.

12. Bhat MG, Jordt RM, Khan MA, Foley CE, Gilbertson TA. Validation of a rat behavioral avoidance model from a drug delivery perspective. Int J Pharm. 2005;303:31-6.

13. Cotterill JV, Massei G, Cowan DP. Masking the taste of the conditioned taste aversion agent levamisole using an ionexchange resin, for practical application in wildlife management. Pest Manag Sci. 2006;62:120-5.

14. Sherman J, Rusiniak KW, Garcia J. Alcohol-ingestive habits: the role of flavor and effect. In: Gallanter M, Begleiter H, Deitrich R, Goodwin D, Gottheil E, Paredes A, et al. editors. Recent developments in alcoholism. New York: Springer; 1984. p. 59-79.

15. Mennella JA, Spector AC, Reed DR, Coldwell SE. The bad taste of medicines: overview of basic research on bitter taste. Clin Ther. 2013;35:1225-46.

16. Picone S, Bedetta M, Paolillo P. Caffeine citrate: when and for how long. A literature review. J Matern Fetal Neonatal Med. 2012;25:11-4.

17. Wu CY, Benet LZ. Predicting drug disposition via application of BCS: transport/absorption/ elimination interplay and development of a biopharmaceutics drug disposition classification system. Pharm Res. 2005;22:11-23.

18. Berridge KC. Measuring hedonic impact in animals and infants: microstructure of affective taste reactivity patterns. Neurosci Biobehav Rev. 2000;24:173-98.

19. Grill HJ, Norgren R. The taste reactivity test. I. Mimetic responses to gustatory stimuli in neurologically normal rats. Brain Res. 1978;143:263-79.

20. Grill HJ, Norgren R. The taste reactivity test. II. Mimetic responses to gustatory stimuli in chronic thalamic and chronic decerebrate rats. Brain Res. 1978;143:281-97.

21. Steiner JE, Glaser D, Hawilo ME, Berridge KC. Comparative expression of hedonic impact: affective reactions to taste by human infants and other primates. Neurosci Biobehav Rev. 2001;25:53-74. 